\title{
Long-term administration of vitamin B12 and adenosine triphosphate for idiopathic sudden sensorineural hearing loss: a retrospective study
}

\author{
Takaomi Kurioka ${ }^{\text {Corresp., } 1}{ }^{,}$Hajime Sano ${ }^{2}$, Shogo Furuki ${ }^{1}$, Taku Yamashita ${ }^{1}$ \\ 1 Department of Otorhinolaryngology, Head and Neck Surgery, Kitasato University, Kanagawa, Japan \\ 2 School of Allied Health Sciences, Kitasato University, Kanagawa, Japan \\ Corresponding Author: Takaomi Kurioka \\ Email address: takaomi@xj9.so-net.ne.jp
}

Background: In idiopathic sudden sensorineural hearing loss (ISSNHL), the relationship between the administration duration of vitamin B12 (vit B12) with adenosine triphosphate (ATP) and their therapeutic effect is not fully understood.

Objective: To investigate the therapeutic effect of long-term ( $\geq 16$ weeks) administration of vit B12 with ATP on the prognosis of ISSNHL patients and compare it with those of short-term ( $<8$ weeks) and middleterm ( $\geq 8$ weeks, $<16$ weeks) administration.

Methods: We retrospectively reviewed the medical records of 117 patients with ISSNHL treated between 2015 and 2018.

Results: The overall recovery rate was 32.5\%. Initial higher hearing threshold and initial higher grade of hearing loss $(\mathrm{HL})$ were associated with a poor prognosis. However, the administration duration of vit B12 and ATP did not influence the overall hearing improvement. With regard to the time course of hearing recovery, there was no significant difference in hearing recovery among the long-, middle-, and shortterm administration groups until 16 weeks after treatment. However, at 16-24 weeks after initial treatment, the short-term administration group exhibited significantly lower hearing recovery than did the long-term administration groups.

Conclusions: The administration duration of vit B12 and ATP did not influence the overall hearing prognosis in ISSNHL, but long-term administration of vit B12 and ATP helped prevent the progression of HL after ISSNHL. Our results suggest that long-term administration of vit B12 and ATP is not necessarily required to treat ISSNHL patients, except for slowly progressing $\mathrm{HL}$ in the affected ears. 
1 Long-term administration of vitamin B12 and adenosine triphosphate for idiopathic sudden sensorineural hearing loss: a retrospective study

5 Takaomi Kurioka M.D., Ph.D. ${ }^{1 *}$ (ORCID:0000-0001-6273-8527)

6 Hajime Sano M.D., Ph.D. ${ }^{2}$ (ORCID:0000-0002-0645-2521)

7 Shogo Furuki M.D. ${ }^{1}$ (ORCID: 0000-0001-5873-8604)

8 Taku Yamashita M.D., Ph.D. ${ }^{1}$ (ORCID:0000-0003-0441-2611)

9

101 Department of Otorhinolaryngology, Head and Neck Surgery, Kitasato University,

11 Kanagawa, Japan

122 Kitasato University, School of Allied Health Sciences, Kanagawa, Japan

$14{ }^{*}$ Correspondence: Takaomi Kurioka

15 Otorhinolaryngology and Head and Neck Surgery, Kitasato University

16 1-15-1 Kitasato, Minami-ku, Sagamihara-shi, Kanagawa 252-0374, Japan

17 TEL: (+81)-42-778-8111

FAX: (+81)-42-778-8441

18 Email address: takaomi@xj9.so-net.ne.jp 


\section{Abstract}

22 Background: In idiopathic sudden sensorineural hearing loss (ISSNHL), the relationship

23 between the administration duration of vitamin B12 (vit B12) with adenosine triphosphate

24 (ATP) and their therapeutic effect is not fully understood.

25 Objective: To investigate the therapeutic effect of long-term ( $\geq 16$ weeks) administration

26 of vit B12 with ATP on the prognosis of ISSNHL patients and compare it with those of

27 short-term (<8 weeks) and middle-term ( $\geq 8$ weeks, $<16$ weeks) administration.

28 Methods: We retrospectively reviewed the medical records of 117 patients with ISSNHL

29 treated between 2015 and 2018.

30 Results: The overall recovery rate was $32.5 \%$. Initial higher hearing threshold and initial

31 higher grade of hearing loss $(\mathrm{HL})$ were associated with a poor prognosis. However, the

32 administration duration of vit B12 and ATP did not influence the overall hearing

33 improvement. With regard to the time course of hearing recovery, there was no significant

34 difference in hearing recovery among the long-, middle-, and short-term administration

35 groups until 16 weeks after treatment. However, at 16-24 weeks after initial treatment,

36 the short-term administration group exhibited significantly lower hearing recovery than did

37 the long-term administration groups.

38 Conclusions: The administration duration of vit B12 and ATP did not influence the overall

39 hearing prognosis in ISSNHL, but long-term administration of vit B12 and ATP helped

40 prevent the progression of $\mathrm{HL}$ after ISSNHL. Our results suggest that long-term

41 administration of vit B12 and ATP is not necessarily required to treat ISSNHL patients,

42 except for slowly progressing HL in the affected ears. 


\section{Introduction}

45 Idiopathic sudden sensorineural hearing loss (ISSNHL) usually occurs as an acute

46 unilateral hearing loss (HL) (Staecker et al. 2019). The exact cause of ISSNHL has not

47 been identified, but several possible etiologies have been proposed, such as vascular

48 dysfunction, neurological disorder, autoimmune disease, or viral infection (Watanabe et

49 al. 2019). Accordingly, the optimal treatment modality for ISSNHL remains controversial,

50 but various comprehensive approaches have been widely adopted, including topical

51 application of steroids, vasodilator, and vitamin supplementation, with systemic steroid

52 therapy being the mainstream treatment (Ahmadzai et al. 2019). However, despite

53 systemic treatment, the spontaneous recovery rate only ranges from $30 \%$ to $60 \%$ (Qiang

54 et al. 2017). Further, although steroid treatment has profound therapeutic benefits, it does

55 not confer therapeutic benefit if the treatment is started more than 2 weeks after onset

56 (Amarillo et al. 2019). After completion of the initial steroid therapy, patients are

57 prescribed various medications such as vitamin supplementation and vasodilator drugs

58 continuously. Particularly, a combination of vitamin B12 (vit B12) and adenosine

59 triphosphate (ATP) is prescribed for ISSNHL patients to improve the auditory neural

60 function and cochlear blood circulation (Ahmadzai et al. 2019).

61 The duration of vit B12 and ATP treatment depends on the period during which the

62 treatment effect of these drugs is sustained. A patient would be willing to take vit B12 and

63 ATP orally for the long term if these medications are likely to provide benefits for a longer

64 period of time. However, the influence of the duration of vit B12 treatment with ATP on

65 hearing prognosis is unclear. Thus, the present study aimed to investigate the influence

66 of treatment duration of vit B12 with ATP on the hearing prognosis of ISSNHL. Towards 
67 this goal, we evaluated the therapeutic effect of long-term ( $\geq 16$ weeks) administration of

68 vit B12 with ATP in comparison to that of short-term ( $<8$ weeks) and middle-term ( $\geq 8$

69 weeks, $<16$ weeks) administration.

71 Materials and methods

\section{Study design and ethical considerations}

73 This retrospective study (B20-056) was approved by the Institutional Review Board of

74 Kitasato University Hospital. The need for informed consent was waived owing to the 75 retrospective nature of the study.

\section{Patients}

78 The subjects were 117 patients with ISSNHL who underwent pure-tone audiometry at Kitasato University Hospital between 2015 and 2018. The inclusion criteria were as

80 follows: (1) sudden sensorineural $\mathrm{HL}$ of $30 \mathrm{~dB}$ or greater in at least three consecutive

81 frequencies; (2) underwent early therapeutic management, that is, treatment was started

82 within 2 weeks after onset; (3) age over 18 years; and (4) follow-up duration longer than

836 months. The exclusion criteria were as follows: (1) age younger than 17 years; (2)

84 middle ear or retro-cochlear pathology; (3) history of Meniere's disease or autoimmune

$85 \mathrm{HL}$; (4) history of genetic or fluctuating HL; (5) history of hearing aid wearing or otologic

86 surgery; and (6) history of intratympanic steroid injection for salvage treatment after

87 systemic steroid administration. 


\section{Assessment of hearing function}

90 Pure-tone audiometry was performed using a conventional device (AA-78; Rion, Tokyo,

91 Japan) in a soundproof room. First, the hearing thresholds were obtained through air

92 conduction $(\mathrm{AC})$ and bone conduction $(\mathrm{BC})$ in frequencies of $0.25,0.5,1,2$, and $4 \mathrm{kHz}$

93 for both ears. To prevent cross-hearing phenomenon causing an erroneous

94 measurement, masking noise was used to occupy the non-test ear while the other ear

95 was tested, as necessary. Briefly, the necessity of masking during the measurement of

96 the AC thresholds was identified based on the minimum interaural attenuation level of 40

$97 \mathrm{~dB}$ for retesting through $\mathrm{AC}$. Meanwhile, in the measurement of $\mathrm{BC}$ thresholds, a masking

98 process was applied using $A B C$ methods (Kurioka et al. 2020c). Thresholds were

99 obtained across all the frequency octaves from $0.25 \mathrm{kHz}$ to $4 \mathrm{kHz}$, and the arithmetic

100 average $\mathrm{AC}$ and $\mathrm{BC}$ thresholds were calculated from the thresholds at $0.25,0.5,1,2$,

101 and $4 \mathrm{kHz}$. The grade of $\mathrm{HL}$, defined according to the Japanese Ministry of Health and

102 Welfare guidelines (Nakashima et al. 2014), was then determined using the initial

103 audiogram data. Hearing recovery was calculated as the difference between average

104 hearing thresholds at different time points, including initial day of treatment and 2,4 , and

1056 months after initial treatment. Siegel's criteria were employed to assess treatment

106 results 6 months after treatment (Table 1), and the patients were accordingly classified

107 into two groups as the recovery group (i.e., complete and partial recovery) and the no 108 recovery group (i.e., slight recovery and no improvement). These two groups were treated

109 with same drug regimen, except for the duration of vit B12 and ATP administration. 


\section{Treatment}

113 All patients were treated with a 10-day course of systemic corticosteroids (betamethasone

$1148 \mathrm{mg}$ via intramuscular injection for the first day followed by betamethasone $4 \mathrm{mg}$ via oral

115 administration for the first 3 days, tapered to $2 \mathrm{mg}$ for the second 3 days, and finally to 1

$116 \mathrm{mg}$ for the last 3 days). Oral administration of vit B12 (1.5 mg daily) and ATP (300 mg

117 daily) was started from initial day of treatment, concurrent with the corticosteroids. To

118 investigate the therapeutic effects of long-term administration of vit B12 and ATP on

119 hearing outcomes in ISSNHL, the patients were divided into three groups according to

120 the administration duration as the short-term ( $<8$ weeks), middle-term ( $\geq 8$ weeks, $<16$

121 weeks) and long-term ( $\geq 16$ weeks) administration groups.

\section{Statistical analyses}

124 The Chi-squared test was used to evaluate clinical characteristics and possible prognostic

125 factors. Student's t-tests and non-parametric Mann-Whitney $U$ tests were used to

126 investigate continuous prognostic factors. For comparisons between more than two

127 groups, a one-way analysis of variance was used followed by Dunn's multiple

128 comparisons for the post hoc test. The parameters that were statistically significant in the

129 univariate analysis were entered into a binary logistic regression model for multivariate

130 analysis. All statistical analyses were performed using GraphPad Prism 8 (GraphPad

131 Software Inc., La Jolla, CA) or JMP 14.2 (SAS Institute Japan Inc., Tokyo, Japan). A p

132 value of $<0.05$ was considered statistically significant. 


\section{Results}

Patient characteristics and treatment outcome

136 The mean age was $58.2 \pm 16.6$ years, and there were 66 and 51 male and female

137 patients, respectively. The patients' clinicodemographic characteristics are presented in

138 Table 2. The mean interval between symptom onset and initial treatment was $4.2 \pm 3.8$

139 days. As accompanying symptoms and complications, 56 patients (47.9\%) had vertigo

140 and 32 patients (27.4\%) had diabetes. The mean hearing threshold at the initial

141 examination was $80.7 \pm 24.0 \mathrm{~dB}$. With respect to initial HL grade, 8 patients had grade 1;

14218 patients, grade 2; 45 patients, grade 3; and 46 patients, grade 4 . The average duration

143 of vit B12 and ATP administration was $4.2 \pm 2.4$ months. With regard to final recovery

144 according to Siegel's criteria, the overall recovery rate (complete + partial recovery) was

$14532.5 \%$. Specifically, $19.7 \%, 12.8 \%, 39.3 \%$, and $28.2 \%$ of the patients achieved complete

146 recovery, partial recovery, slight recovery, and no improvement, respectively (Table 1).

\section{Prognostic factors}

149 In univariate analysis, the initial hearing threshold and initial grade of HL was significantly

150 lower in the recovery group than that in the no recovery group $(p<0.0001)$. With regard

151 to the associated symptoms, there was a significantly higher incidence of vertigo in the

152 no recovery group than in the recovery group $(p=0.04)$. The other variables, including

153 age, sex, days to initiation of treatment, and diabetes were not significantly different

154 between the two groups. The duration of vit B12 and ATP administration was slightly 
155 longer in the no recovery group than that in the recovery group, but the difference was

156 not significant. The significant variables in the univariate analysis were included in the

157 multivariate analysis. The results showed that higher initial hearing threshold and higher

158 initial grade of HL were associated with a poor prognosis in ISSNHL patients (Table 3).

\section{Administration duration of vit B12 and ATP}

161 The short-term, middle-term, and long-term administration groups involved 15, 41, and

16238 patients, respectively. The patients with complete recovery within 24 weeks after onset

$163(n=23)$ were excluded because the vit B12 and ATP administration was terminated

164 following the confirmation of complete recovery. Specifically, the duration of drug

165 administration was shortened due to complete recovery, but the shortened administration

166 did not contribute to complete recovery. Therefore, it was not appropriate to include the

167 patients with complete recovery within 24 weeks after onset for the analysis of the effect

168 of drug administration duration. In this study, the duration of administration was

169 determined by the patients after consultation with their physician, even though they did

170 not achieve complete recovery. The clinical features of each group are presented in Table

171 4. The mean duration of drug administration in the short, middle, and long-term groups

172 was $1.9,3.3$, and 6.3 months, respectively. In univariate analysis, the duration of drug

173 administration was significantly different among the groups $(p<0.0001)$, but the clinical

174 characteristics and hearing results were comparable $(p>0.05)$. This indicated that the

175 duration of drug administration was not influenced by the patient's clinical characteristics

176 and did not affect the overall hearing outcome. Ultimately, these results show that long- 
177 term administration of vit B12 and ATP had no significant impact on the hearing prognosis

178 within our observation period.

179 We also investigated the time course of hearing recovery in each group. As shown in

180 Figure $1 \mathrm{~A}$, the thresholds $(\mathrm{dB})$ of total hearing recovery within 24 months after symptom

181 onset in the short-, middle-, and long-term administration groups were $18.9 \pm 5.8,22.4 \pm$

1822.3 , and $22.7 \pm 3.0$, respectively, with no significant differences $(p=0.76)$. The time

183 course of hearing recovery in the overall population was $18.9 \pm 1.7,3.1 \pm 0.7$, and $0.04 \pm$

1840.4 within $0-2,2-4$, and $4-6$ months after symptom onset, respectively $(p<0.0001$; Fig.

185 1B). Hearing recovery within the initial 2 months after symptom onset was significantly

186 higher than that within $2-4$ months $(p<0.0001)$ and $4-6$ months $(p<0.0001)$. Moreover,

187 hearing recovery within $2-4$ months after symptom onset was significantly higher than

188 that within $4-6$ months $(p=0.04)$. This result indicated that hearing recovery in the initial

1892 months after onset is higher and that the probability of recovery decreases as the

190 duration after symptom onset increases. The time course of hearing recovery of both the

191 affected and contralateral ears in the three groups are shown in Figure 2. There was no

192 significant difference in hearing recovery among the three groups until 16 weeks after

193 onset. However, hearing recovery of the affected ears was significantly lower in the short-

194 term administration group than that in the long-term administration group at 16-24 weeks

195 after symptom onset $(p=0.03)$. Meanwhile, there was no significant difference in hearing

196 recovery of the contralateral ears among the three groups throughout the study period.

197 This indicated a slow progression of $\mathrm{HL}$ in the affected ear and that long-term

198 administration of vit B12 and ATP might prevent progressive HL in the affected ear in

199 ISSNHL. We performed an additional analysis of hearing recovery at each frequency. As 
200 shown in Figure 3, hearing recovery appeared to be greater at 0.5 and $1.0 \mathrm{kHz}$ than that

201 at 2.0 and $4.0 \mathrm{kHz}$ until 8 weeks after onset. In addition, significant differences between

202 long-term and short-term drug administration were observed at only $2.0 \mathrm{kHz}$ during the 203 4-6 months after the onset of ISSNHL.

\section{Discussion}

206 The influence of the duration of vit B12 and ATP treatment on hearing prognosis has been

207 unclear. In our study, the administration periods of vit B12 and ATP were not prognostic 208 factors. However, this result does not necessarily indicate that long-term administration 209 of vit B12 and ATP has no therapeutic effect on the overall prognosis of ISSNHL as 210 hearing recovery was significantly lower in the short-term administration group than that

211 in the middle and long-term administration groups within 4-6 months after onset. This

212 indicates that vit B12 and ATP treatment might have gradual therapeutic effects on

213 preventing the deterioration in hearing rather than promoting the hearing recovery.

214 Hearing recovery within the initial 2 months was also significantly higher than that in the

215 later phase, indicating that early initial treatment within 2 months after symptom onset is 216 extremely important.

217 The optimal treatment strategy for ISSNHL has not been established to date because the 218 cause and etiology of ISSNHL remain unclear. However, circulation and neurological

219 disorders are thought to be one of the major causes for ISSNHL (Hsu et al. 2016).

220 Because the cochlea is supplied by the labyrinthine artery, which has no collateral 221 circulation, the obstruction of blood supply by thrombosis or hemorrhage may cause 
222 cochlear damages that results in ISSNHL. Diabetes, hyperlipidemia, and aging are also

223 well-known factors of microvascular disease that results in blood circulation disorders in

224 the inner ear (Akinpelu et al. 2014; Orita et al. 2007). Various empirical treatments have

225 been applied to improve blood circulation and to increase oxygen supply to the inner ear,

226 including vasodilators, steroids, plasma expanders, and anticoagulant agents (Xie et al.

227 2018). Particularly, systemic steroids have become the most commonly used treatment

228 for ISSNHL patients, with the dose tapered within 2 weeks (Slattery et al. 2005). The use

229 of intra-tympanic steroid injection for the treatment of ISSNHL has also recently increased

230 owing to its comparable effects to systemic steroids and the additional benefit of salvage

231 treatment (Kordis \& Battelino 2017). After the initial steroid treatment, which usually lasts

232 for 1-2 weeks, patients are generally prescribed vit B12 and ATP supplementation for

233 further management.

234 Vitamin B12 plays an important role in the maintenance of normal neural function (Altun

235 \& Kurutas 2016). Accordingly, vit B12 deficiency leads to anemia, demyelination, axonal

236 degeneration, and, ultimately, neuronal loss. In the auditory system, vit B12 deficiency is

237 known to have negative effects on hearing by affecting myelinization of the auditory

238 neurons at the retro-cochlear region. Additionally, auditory neural degenerations, such as

239 disruption of cochlear synapses, demyelination, and shrinkage of auditory nerves,

240 gradually progress after decreased auditory inputs (Kurioka et al. 2020a; Kurioka et al.

241 2020b). Vitamin B12 supplementation has been shown to increase the number of

242 Schwann cells and myelinated nerve fibers and the diameter of the axons, thereby

243 promoting the regeneration of myelinated nerve fibers and the proliferation of Schwann

244 cells (Lopatina et al. 2011). Therefore, supplementary vit B12 treatment is considered in 
245 auditory diseases to prevent neural disorders and to enhance neurovascular endothelial

246 function (Singh et al. 2016). Vitamin B12 supplementation also reduces homocysteine

247 synthesis, which is a vascular and thrombotic risk factor and causes vascular injury by

248 reducing the amount of nitric oxide (NO). The reduced homocysteine synthesis leads to

249 vasodilatation as a result of an increase in the amount of NO (Toda \& Okamura 2016).

250 Thus, vit B12 can cause an increase in vascular perfusion and cellular metabolism in the

251 cochlea.

252 ATPs are often used as vasodilators to increase cochlear blood flow in auditory

253 diseases. The vasodilator effect of ATP is mediated by the endothelium and follows the

254 release of NO. A previous study reported that treatment with ATP resulted in a large

255 increase in cochlear blood flow and a decrease in blood pressure owing to the vasodilator

256 actions of ATP (Munoz et al. 1999). Another study reported that a $300 \mathrm{mg}$ dose of ATP

257 has therapeutic effects for inner ear pathologies, such as Meniere's disease (Mizukoshi

258 et al. 1983). Thus, ATP and vit B12 are widely used for the treatment of ISSNHL owing

259 to their various biological therapeutic effects.

260 The prognosis for recovery from ISSNHL can be influenced by various factors, including 261 age, accompanying symptoms such as vertigo, degree of $\mathrm{HL}$, audiometric configuration,

262 and time to treatment initiation (Kim et al. 2018; Kuhn et al. 2011; Lin et al. 2016). The

263 findings of the present study indicated that the initial hearing threshold and initial grade

264 of $\mathrm{HL}$ were useful prognostic factors, consistent with the findings of a previous study

265 (Kuhn et al. 2011). With respect to the treatment period after ISSNHL onset, it has been

266 reported that hearing remained relatively stable after a period of 2-3 months from

267 symptom onset (Kallinen et al. 2001; Kanzaki et al. 1988; Psifidis et al. 2006). It is possible 
268 that the period of 2 months could be consistent with the natural history of the disease, 269 regardless of which therapeutic strategy is applied. Thus, any additional treatment after

2702 months should not affect the outcome of the hearing, consistent with our results.

271 However, a long-term follow-up study reported that $9.9 \%$ patients showed an

272 improvement over a period of 3 months after the onset of ISSNHL (Yeo et al. 2007).

273 Nevertheless, one study found that $25 \%$ of patients had long-term hearing deterioration

274 after the onset of ISSNHL (Furuhashi et al. 2002). This is consistent with our results that

275 ISSNHL affected the involved ear more gradually than the contralateral ears. This hearing

276 deterioration appeared to have no relationship with age-related $\mathrm{HL}$ because the

277 deterioration was observed only in the affected ears in this study. Although the overall

278 hearing recovery did not differ according to the duration of administration, we found some

279 benefit for the prevention of hearing deterioration with long-term administration. However,

280 these results generally do not indicate that long-term vit B12 and ATP should be adopted

281 for ISSNHL patients. Furthermore, it is generally believed that hearing recovery would

282 differ between acute low-tone sensorineural HL and ISSNHL. Therefore, the initial type

283 of $\mathrm{HL}$ in ISSNHL might be associated with delayed changes in hearing after the onset.

284 Additional studies with a longer observation period are needed to elucidate the accurate

285 therapeutic effects of vit B12 and ATP in ISSNHL.

286 This study has some limitations. First, this was a retrospective study conducted in a single

287 hospital, and the sample size was relatively small. Second, the duration of vit B12 and

288 ATP treatment in this study was influenced by various factors, such as patient preferences

289 and drug therapeutic effects. However, despite these limitations, we believe that our study

290 is valuable because to the best of our knowledge, this is the first to focus on the 
291 relationship between the duration of vit B12 and ATP administration and prognosis in 292 ISSNHL. Our findings have important clinical implications in the understanding and 293 management of patients with ISSNHL. Although the underlying therapeutic mechanisms 294 of vit B12 and ATP in ISSNHL are not well known, its effects need to be considered in the 295 management of ISSNHL in the clinic. Further prospective studies with larger populations 296 are needed to validate our findings.

298 Conclusions

299 The administration duration of vit B12 and ATP did not influence the hearing prognosis in 300 ISSNHL. Higher initial hearing threshold and higher initial grade of HL were associated 301 with a poor prognosis. Compared to the contralateral ear, HL progressed gradually in the 302 affected ear. Long-term administration of vit B12 and ATP might help prevent progressive $303 \mathrm{HL}$ in the affected ear. 
305

306

307

308

309

310

311

312

313

314

322

323

324

325

326

327

328

329

330

\section{References}

Ahmadzai N, Kilty S, Cheng W, Esmaeilisaraji L, Wolfe D, Bonaparte JP, Schramm D, Fitzpatrick E, Lin V, Skidmore B, Moher D, and Hutton B. 2019. A systematic review and network meta-analysis of existing pharmacologic therapies in patients with idiopathic sudden sensorineural hearing loss. PLoS One 14:e0221713. 10.1371/journal.pone.0221713

Akinpelu OV, Ibrahim F, Waissbluth S, and Daniel SJ. 2014. Histopathologic changes in the cochlea associated with diabetes mellitus--a review. Otol Neurotol 35:764-774. 10.1097/MAO.0000000000000293

Altun I, and Kurutas EB. 2016. Vitamin B complex and vitamin B12 levels after peripheral nerve injury. Neural Regen Res 11:842-845. 10.4103/1673-5374.177150

Amarillo E, Navarro A, Hernandez-Garcia E, and Plaza G. 2019. Intratympanic steroids for combined treatment of idiopathic sudden hearing loss: when is it too late? Acta Otolaryngol 139:632-635. 10.1080/00016489.2019.1614222

Furuhashi A, Matsuda K, Asahi K, and Nakashima T. 2002. Sudden deafness: long-term follow-up and recurrence. Clin Otolaryngol Allied Sci 27:458-463. 10.1046/j.13652273.2002.00612.x

Hsu YH, Hu HY, Chiu YC, Lee FP, and Huang HM. 2016. Association of Sudden Sensorineural Hearing Loss With Vertebrobasilar Insufficiency. JAMA Otolaryngol Head Neck Surg 142:672-675. 10.1001/jamaoto.2016.0845

Kallinen J, Laurikainen E, Bergroth L, and Grenman R. 2001. A follow-up study of patients suffering from sudden sensorineural hearing loss. Acta Otolaryngol 121:818-822. $10.1080 / 00016480152602267$

Kanzaki J, Taiji H, and Ogawa K. 1988. Evaluation of hearing recovery and efficacy of steroid treatment in sudden deafness. Acta Otolaryngol Suppl 456:31-36. 10.3109/00016488809125074 
331 Kim JY, Han JJ, Sunwoo WS, Koo JW, Oh SH, Park MH, and Kim YH. 2018. Sudden

332

333

334

335

336

337

338

339

340 sensorineural hearing loss in children and adolescents: Clinical characteristics and age-related prognosis. Auris Nasus Larynx 45:447-455. 10.1016/j.anl.2017.08.010

Kordis S, and Battelino S. 2017. The Role of High Dose Intratympanic Dexamethasone as Salvage Therapy for Idiopathic Sudden Sensorineural Hearing Loss. J Int Adv Otol 13:318-321. 10.5152/iao.2017.3896

Kuhn M, Heman-Ackah SE, Shaikh JA, and Roehm PC. 2011. Sudden sensorineural hearing loss: a review of diagnosis, treatment, and prognosis. Trends Amplif 15:91105. $10.1177 / 1084713811408349$

Kurioka T, Mogi S, Tanaka M, and Yamashita T. 2020a. Activity-Dependent Neurodegeneration and Neuroplasticity of Auditory Neurons Following Conductive Hearing Loss in Adult Mice. Cell Mol Neurobiol. 10.1007/s10571-020-00829-y

Kurioka T, Mogi S, and Yamashita T. 2020b. Transient Conductive Hearing Loss Regulates Cross-Modal VGLUT Expression in the Cochlear Nucleus of C57BL/6 Mice. Brain Sciences 10:260.

Kurioka T, Sano H, Furuki S, and Yamashita T. 2020c. Effects of the Conductive Component of Hearing Loss on Speech Discrimination Ability. J Int Adv Otol. 10.5152/iao.2020.7870

Lin CF, Lee KJ, Yu SS, and Lin YS. 2016. Effect of comorbid diabetes and hypercholesterolemia on the prognosis of idiopathic sudden sensorineural hearing loss. Laryngoscope 126:142-149. 10.1002/lary.25333

Lopatina T, Kalinina N, Karagyaur M, Stambolsky D, Rubina K, Revischin A, Pavlova G, Parfyonova Y, and Tkachuk V. 2011. Adipose-derived stem cells stimulate regeneration of peripheral nerves: BDNF secreted by these cells promotes nerve healing and axon growth de novo. PLoS One 6:e17899. 10.1371/journal.pone.0017899

Mizukoshi K, Watanabe Y, Watanabe I, Okubo J, Matsunaga T, Matsunaga T, Takayasu S, Kato I, and Tanaka T. 1983. Subjective and objective evaluation of medical 
359

treatment for Meniere's disease, with special reference to the dose response for adenosine triphosphate. Adv Otorhinolaryngol 30:355-361. 10.1159/000407674

Munoz DJ, McFie C, and Thorne PR. 1999. Modulation of cochlear blood flow by extracellular purines. Hear Res 127:55-61. 10.1016/s0378-5955(98)00161-0

Nakashima T, Sato H, Gyo K, Hato N, Yoshida T, Shimono M, Teranishi M, Sone M, Fukunaga Y, Kobashi G, Takahashi K, Matsui S, and Ogawa K. 2014. Idiopathic sudden sensorineural hearing loss in Japan. Acta Otolaryngol 134:1158-1163. 10.3109/00016489.2014.919406

Orita S, Fukushima K, Orita Y, and Nishizaki K. 2007. Sudden hearing impairment combined with diabetes mellitus or hyperlipidemia. Eur Arch Otorhinolaryngol 264:359-362. 10.1007/s00405-006-0196-6

Psifidis AD, Psillas GK, and Daniilidis J. 2006. Sudden sensorineural hearing loss: longterm follow-up results. Otolaryngol Head Neck Surg 134:809-815. 10.1016/j.otohns.2005.12.002

Qiang Q, Wu X, Yang T, Yang C, and Sun H. 2017. A comparison between systemic and intratympanic steroid therapies as initial therapy for idiopathic sudden sensorineural hearing loss: a meta-analysis. Acta Otolaryngol 137:598-605. 10.1080/00016489.2016.1260157

Singh C, Kawatra R, Gupta J, Awasthi V, and Dungana H. 2016. Therapeutic role of Vitamin B12 in patients of chronic tinnitus: A pilot study. Noise Health 18:93-97. 10.4103/1463-1741.178485

Slattery WH, Fisher LM, Iqbal Z, and Liu N. 2005. Oral steroid regimens for idiopathic sudden sensorineural hearing loss. Otolaryngol Head Neck Surg 132:5-10. 10.1016/j.otohns.2004.09.072

Staecker H, Jokovic G, Karpishchenko S, Kienle-Gogolok A, Krzyzaniak A, Lin CD, Navratil P, Tzvetkov V, Wright N, and Meyer T. 2019. Efficacy and Safety of AM-111 in the Treatment of Acute Unilateral Sudden Deafness-A Double-blind, Randomized, 
Placebo-controlled Phase 3 Study. Otol Neurotol 40:584-594.

388 Toda N, and Okamura T. 2016. Hyperhomocysteinemia impairs regional blood flow:

389 involvements of endothelial and neuronal nitric oxide. Pflugers Arch 468:1517-1525.

$390 \quad 10.1007 / \mathrm{s} 00424-016-1849-y$

391 Watanabe H, Sano H, Maki A, Ino T, Nakagawa T, Okamoto M, and Yamashita T. 2019.

392 Investigation of Stress Levels before the Onset of Idiopathic Sudden Sensorineural

393 Hearing Loss. J Int Adv Otol 15:51-55. 10.5152/iao.2019.6197

394 395

Xie S, Qiang Q, Mei L, He C, Feng Y, Sun H, and Wu X. 2018. Multivariate analysis of prognostic factors for idiopathic sudden sensorineural hearing loss treated with adjuvant hyperbaric oxygen therapy. Eur Arch Otorhinolaryngol 275:47-51. $10.1007 / \mathrm{s} 00405-017-4784-4$

Yeo SW, Lee DH, Jun BC, Park SY, and Park YS. 2007. Hearing outcome of sudden sensorineural hearing loss: long-term follow-up. Otolaryngol Head Neck Surg 136:221-224. 10.1016/j.otohns.2006.10.021 
Figure 1

Overall hearing recovery according to the duration of vit B12 and ATP administration (A) and in the overall population (B).

(A) The total hearing threshold recovery from symptom onset to 6 months was comparable among the three groups. (B) Hearing recovery in the initial 2 months was significantly higher than that in the later periods. Hearing recovery within 2-4 months after symptom onset was also significantly higher than that at 4-6 months after onset. $p$ values are indicated as $* * p<$ $0.01, * * * * p<0.0001$.

A

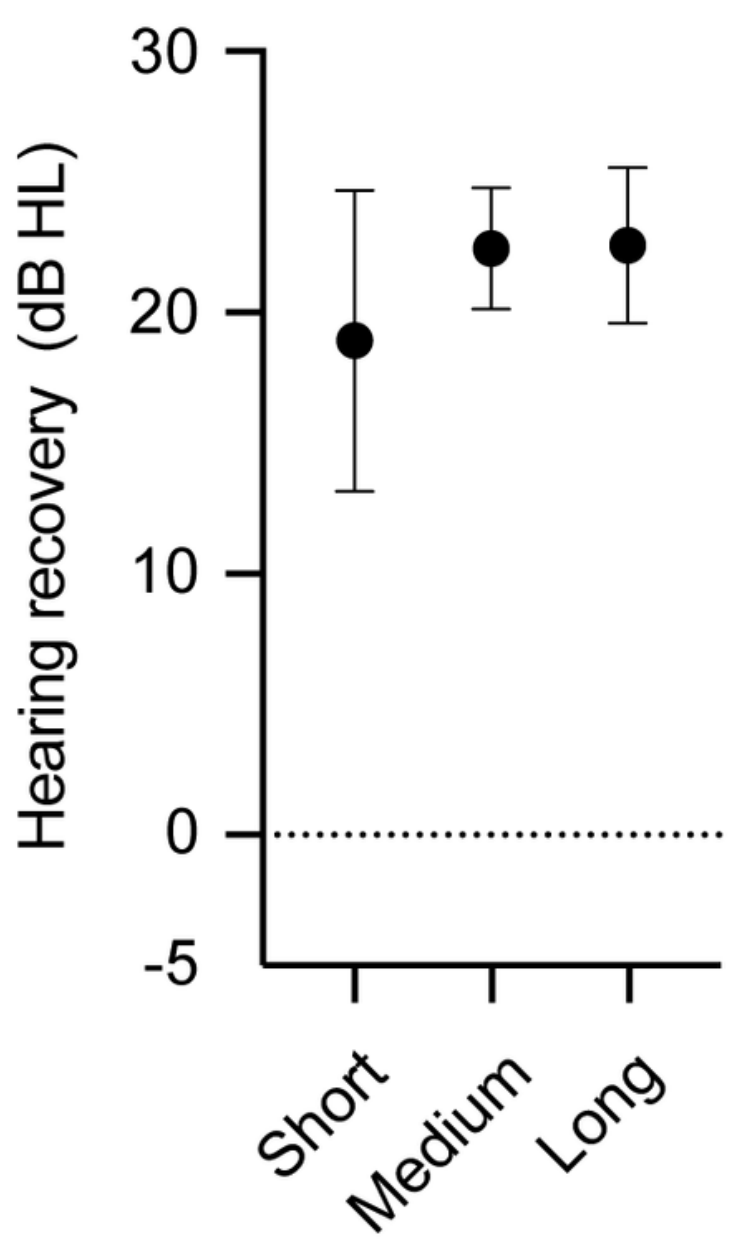

B

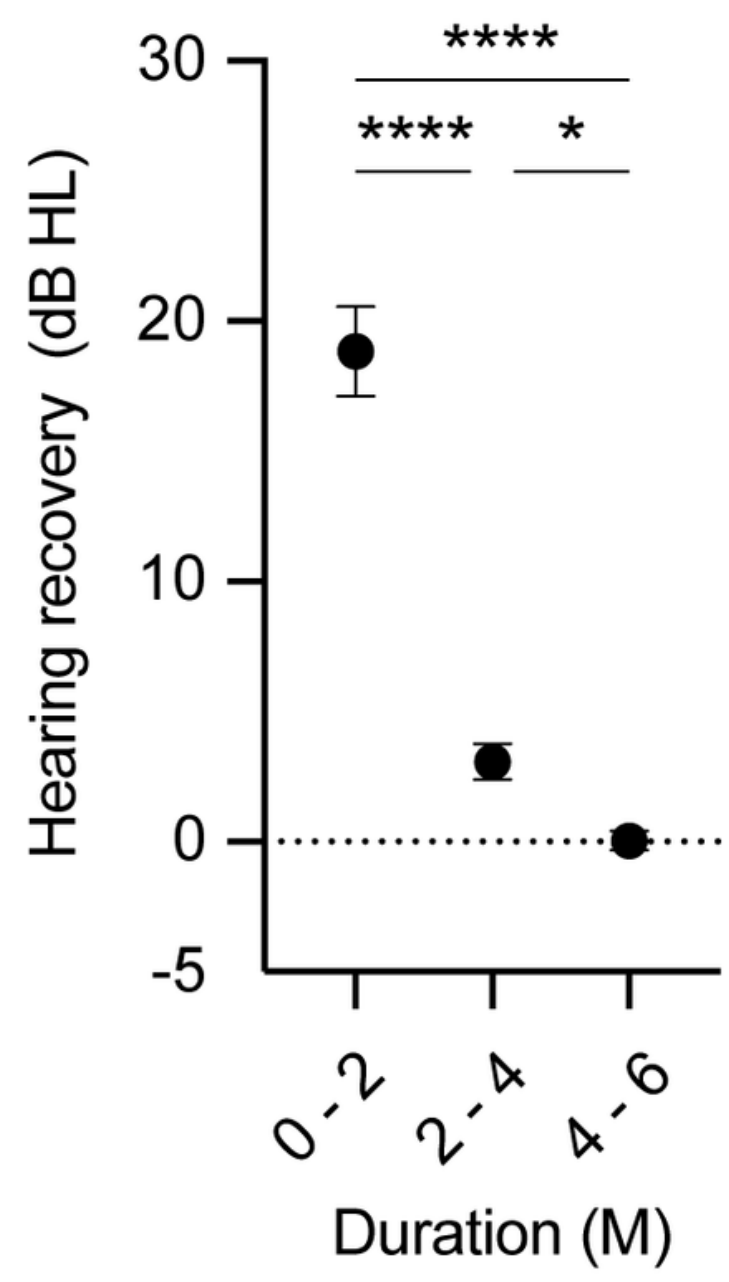




\section{Figure 2}

Time course of hearing recovery in the affected ears and contralateral ears.

Hearing recovery in the affected ears was comparable among the three groups within 0-2 and 2-4 months after symptom onset. Hearing recovery in the short-term administration group (black) was significantly lower than that in long-term administration groups (red) at 4-6 months after symptom onset. In the contralateral ears, there were no significant differences in hearing recovery among the three groups across all time points after symptom onset. $p$ values are indicated as $* p<0.05$.

\section{Affected ear}

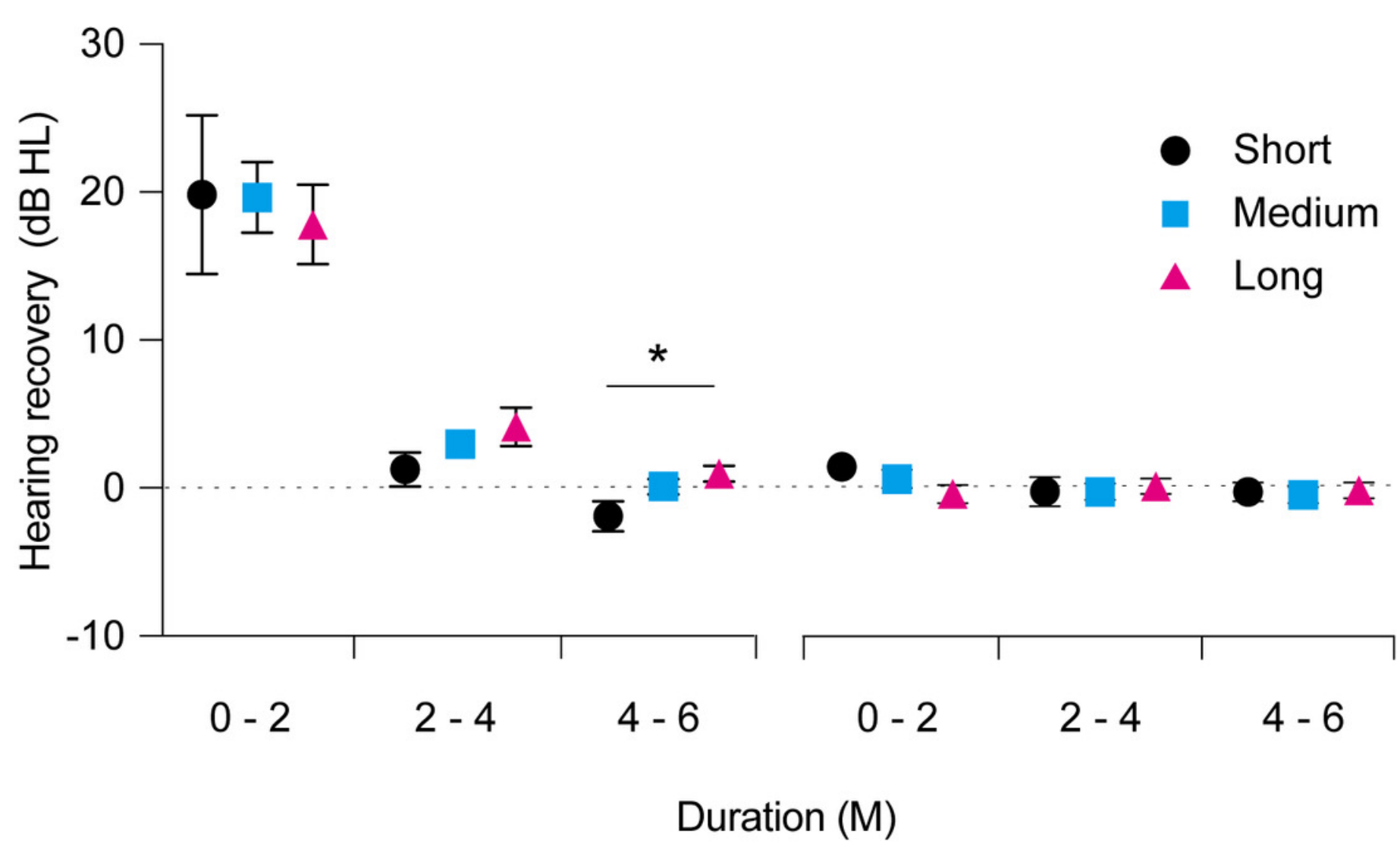


Figure 3

Time course of hearing recovery in the affected ears at each frequency over the $0.5-4.0$ $\mathrm{kHz}$ range.

Hearing recovery in the affected ears was comparable among the three groups within 0-2 and 2-4 months after onset. Hearing recovery differed significantly between the short-term (black) and long-term administration groups (red) at 4-6 months after onset only at $2.0 \mathrm{kHz}$ (C). P values are indicated as $* p<0.05$. 

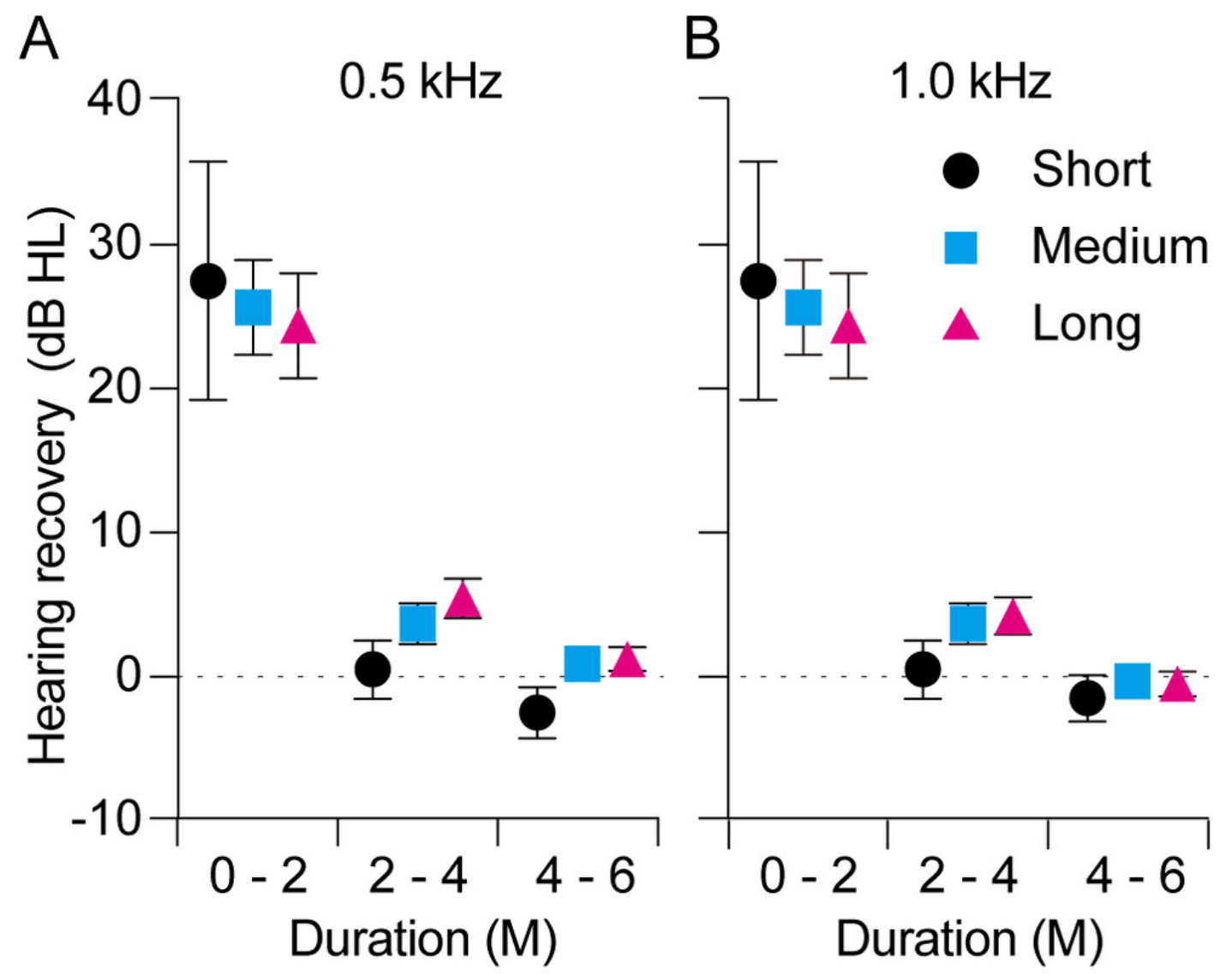

C
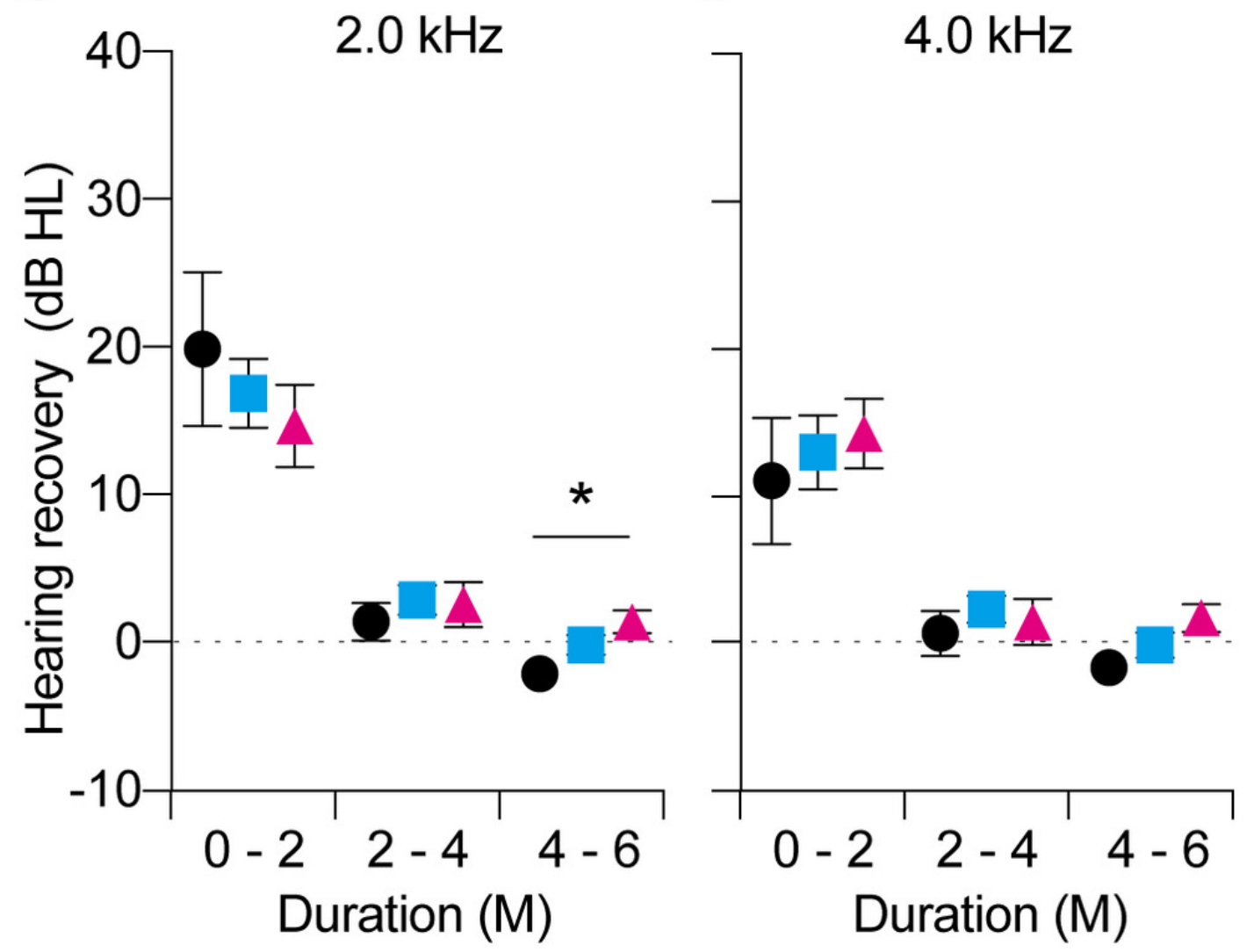

Peer) reviewing PDF | (2020:06:50084:1:1:NEW 30 Oct 2020) 
Table $\mathbf{1}$ (on next page)

Final outcomes according to Siegel's criteria 


\section{Table 1.}

\begin{tabular}{lc}
\hline Description & $\mathrm{n}(\%)$ \\
\hline Complete recovery: final hearing level $<25 \mathrm{~dB}$ & $23(19.7)$ \\
Partial recovery: final hearing level $25-45 \mathrm{~dB}$ with hearing gain $\geq 15 \mathrm{~dB}$ & $15(12.8)$ \\
Slight recovery: final hearing $>45 \mathrm{~dB}$ with hearing gain $\geq 15 \mathrm{~dB}$ & $46(39.3)$ \\
No recovery: hearing gain $\leq 15 \mathrm{~dB}$ & $33(28.2)$ \\
\hline
\end{tabular}


Table 2 (on next page)

Clinicodemographic patient characteristics 


\section{Table 2.}

\begin{tabular}{lc}
\hline Variables & Value \\
\hline Number of patients & 117 \\
Age (years) & $58.2 \pm 16.6$ \\
Sex (male/female) & $66 / 51$ \\
Initiation of treatment (days) & $4.2 \pm 3.8$ \\
Vertigo (+/-) & $56 / 61$ \\
Diabetes (+/-) & $32 / 85$ \\
Initial hearing level (dB) & $80.7 \pm 24.0$ \\
Initial grade of HL (1/2/3/4) & $8 / 18 / 45 / 46$ \\
Duration of vit B12 and ATP administration (months) & $4.2 \pm 2.4$ \\
\hline
\end{tabular}

2 
Table 3 (on next page)

Prognostic factors of ISSNHL 
1 Table 3.

\begin{tabular}{lcccc}
\hline & Recovery & No recovery & Univariate & Multivariate \\
\cline { 5 - 5 } & group & group & & \\
& $(\mathrm{n}=38)$ & $(\mathrm{n}=79)$ & $p$ & $p$ \\
\hline Age (years) & $55.6 \pm 15.5$ & $59.5 \pm 17.0$ & 0.23 & \\
Sex (male/female) & $17 / 21$ & $49 / 30$ & 0.08 & \\
Time to initiation of treatment (days) & $4.3 \pm 3.0$ & $4.2 \pm 4.2$ & 0.90 & \\
Vertigo (+/-) & $13 / 25$ & $43 / 36$ & $0.04^{*}$ & 0.27 \\
Diabetes (+/-) & $12 / 26$ & $22 / 57$ & 0.68 & \\
Initial hearing level (dB) & $65.9 \pm 17.7$ & $87.9 \pm 23.4$ & $<0.0001^{* * * *}$ & $0.001^{* *}$ \\
Initial grade of HL (1/2/3/4) & $3 / 11 / 22 / 2$ & $5 / 7 / 23 / 44$ & $<0.0001^{* * * *}$ & $0.03^{*}$ \\
Duration of vit B12 and ATP & $3.7 \pm 2.6$ & $4.4 \pm 2.2$ & 0.11 & \\
administration (months) & & & & \\
\hline
\end{tabular}

2 


\section{Table 4 (on next page)}

Clinicodemographic patient characteristics according to the duration of vit B12 and ATP administration 
$1 \quad$ Table 4.

\begin{tabular}{lcccc}
\hline & $\begin{array}{c}\text { Short } \\
(\mathrm{n}=15)\end{array}$ & $\begin{array}{c}\text { Medium } \\
(\mathrm{n}=41)\end{array}$ & $\begin{array}{c}\text { Long } \\
(\mathrm{n}=38)\end{array}$ & $p$ \\
\hline Duration of vit B12 and ATP & $1.9 \pm 0.4$ & $3.3 \pm 0.9$ & $6.3 \pm 1.8$ & $<0.0001^{\text {****}}$ \\
administration (months) & $65.7 \pm 16.6$ & $60.2 \pm 14.4$ & $56.4 \pm 17.7$ & 0.17 \\
Age (years) & $12 / 3$ & $20 / 21$ & $24 / 14$ & 0.09 \\
Sex (male/female) & $4.7 \pm 6.1$ & $3.9 \pm 3.1$ & $4.4 \pm 4.3$ & 0.78 \\
Time to initiation of treatment (days) & $7 / 8$ & $19 / 22$ & $21 / 17$ & 0.70 \\
Vertigo (+/-) & $3 / 12$ & $14 / 27$ & $11 / 27$ & 0.59 \\
Diabetes (+/-) & $87.7 \pm 23.8$ & $80.1 \pm 22.7$ & $89.2 \pm 22.9$ & 0.19 \\
Initial hearing level (dB) & $1 / 1 / 4 / 9$ & $2 / 7 / 17 / 15$ & $2 / 4 / 11 / 21$ & 0.62 \\
Initial grade of HL (1/2/3/4) & $68.8 \pm 29.8$ & $57.8 \pm 21.3$ & $66.5 \pm 20.7$ & 0.14 \\
Final hearing level (dB) & $3 / 5 / 7$ & $10 / 19 / 12$ & $2 / 22 / 14$ & 0.13 \\
Hearing improvement (Type 2/3/4) & & & & \\
\hline
\end{tabular}

2 